\title{
Testicular Tumour with Inguinal Nodal Metastasis - A Rare Presentation
}

\author{
D Suresh Kumar ${ }^{1}$, TD Balamurugan ${ }^{1 *}$, MP Vishwanathan ${ }^{1}$, S Sivakumari ${ }^{1}$, \\ Kavitha Sukumar ${ }^{1}$ Navin Naushad ${ }^{1}$, Shakthi Usha Devi ${ }^{1}$ \\ ${ }^{1}$ Dept of Surgical Oncology, Tamilnadu Government Multi Super Specialty Hospital, \\ Madras Medical College, Chennai, India.
}

\begin{abstract}
:
Introduction: The lymphatics of testis drains through gonadal vessels to retroperitoneal lymph nodes. Hence they do not metastasize to inguinal lymph nodes. We report a case of testicular tumour with inguinal nodal metastasis and management of the same. We discuss the pathophysiology, complications and preventive measures along with literary review.

Case report: A 31 year male underwent radical orchidectomy for right testicular tumour (Non Seminomatous Germ cell tumour). After six months, he recurred with ipsilateral metastasic inguinal lymph nodal mass with elevated serum tumor markers. After 4 cycles of chemotherapy, radical inguinal block dissection was done for residual nodes.

Discussion: Inguinal lymph nodal spread of testicular tumor makes it a metastatic disease (Mla). It rarely occurs with an intact scrotal barrier. Various mechanisms have been postulated for such presentation. 2\% of patients with testicular tumour develop inguinal metastasis. It is commonly associated with non seminomatous germ cell tumour. The risk increases in patients who have had the lymphatics disrupted by prior inguinal or scrotal surgery.

Conclusion: In any suspicious testicular tumour, radical inguinal orchidectomy with high ligation of cord is warranted. This is to prevent lymphatic disruption by scrotal orchidectomy and risk of inguinal nodal metastasis. We have reported a case of inguinal nodal metastasis with intact scrotal barrier and discussed its mechanism, complications and prevention with literary review of similar cases.
\end{abstract}

Keywords:- Testicular tumour,Inguinal nodal metastasis,Radical Inguinal orchidectomy(RIO) ,Radical Inguinal Block Dissection(RIBD).

\section{Introduction}

Case Report: A 31 year male was evaluated elsewhere for painful swelling in right hemiscrotum. He was detected to have Right testicular mass and underwent a right Radical Inguinal Orchiectomy(RIO). Details of upfront Serum Tumour markers and imaging were not available, hence clinical stage was not known. On histopathological examination, it was found to be a Mixed Germ cell tumour(Yolk sac +Teratoma components). The patient defaulted further management. Six months later he developed a swelling in the right inguinal region, which gradually progressed over 2 months and patient presented to our centre.

No similar complaints in the left side. No chest/abdominal symptoms, No h/o congenital anomaly of testis. No h/o past surgery in inguinal/scrotal region. No significant family history. He was a chronic alcoholic. He is married with two children. No comorbidities.

On examination, Performance status: ECOG-1, not pale, not icteric, no generalised lymphadenopathy and no gynaecomastia. On local examination of Right Inguino scrotal region, a $15 \times 12 \times 6 \mathrm{~cm}$ mass in right inguinal region extending to right hemiscrotum and just crossing midline. Scar(of past RIO) noticed, skin is warm and adherent with the mass, the mass has restricted mobility, not fixed to underlying structures. Right external iliac and para aortic nodes are clinically not palpable. No distal limb lymphedema. Contralateral side had 2-3 inguinal nodes palpable, largest $1.5 \times 1 \mathrm{~cm}$. Left testicle was clinically normal.No left supra clavicular node. Chest \& Abdomen Clinically showed no evidence of metastasis.

\section{Investigations}

\begin{tabular}{|l|l|l|}
\hline Serum tumor markers & Test & Normal value \\
\hline AFP $(\mathrm{ng} / \mathrm{ml})$ & 1405 & Upto 0.8 \\
\hline Beta HCG & 187.5 & Upto 5 \\
\hline LDH(U/L) $(\mathrm{mIU} / \mathrm{ml})$ & 1093 & Upto 480 \\
\hline
\end{tabular}


CECT Abdomen: Conglomerate of Bulky right inguinal nodes measuring $15 \times 12 \times 5 \mathrm{~cm}$, moderately enhancing with areas of central necrosis and extending to right hemiscrotum. No iliac, retroperitoneal or para aortic nodes. Few insignificant left inguinal nodes present. Solid abdominal organs were normal, no free fluid. CT Chest: Normal study

Stage: c Tx N0 M1a S2 --- Stage IIIB

Tumour Board Policy: In view of elevated Serum Tumour markers, advised 4 cycles of Chemotherapy with BEP regimen (Bleomycin, Etoposide and Cisplatin)

On Post chemo reassessment:

i) Clinically,

Inguinal mass size $12 \times 10 \times 4 \mathrm{~cm}$ (a decrease by $4 \mathrm{~cm}$ ), focus of skin ulceration present, no evidence disease in new sites.

ii) Biochemically,

\begin{tabular}{|l|l|l|l|}
\hline Sr. Tumour markers & Pre Chemo & Post Chemo & Ref.Values \\
\hline AFP & 1405 & 8.7 & Upto $0.8 \mathrm{ng} / \mathrm{ml}$ \\
\hline Beta HCG & 187.5 & 0.1 & Upto $5 \mathrm{mIU} / \mathrm{ml}$ \\
\hline LDH & 1093 & 501 & Upto $480 \mathrm{U} / \mathrm{L}$ \\
\hline
\end{tabular}

iii) Imaging-CECT Abdomen: Right inguinal nodal mass $12 \times 9 \times 4 \mathrm{~cm}$ with extension to right hemiscrotum,skin invasion present. No nodes in iliac, para aortic region.

CT Chest-NAD

Post Chemo Stage : y Tx N0 M1a S1 -Stage IIIA (Down staged from IIIB). Post chemotherapy reassessment infers, the tumour markers were normalized and biochemically the tumour is inert but there is poor responding residual inguinal nodes with skin involvement. In view of Non responding Residual Inguinal nodes, Surgical management was contemplated.

Surgery:Right Radical Inguinal Block Dissection (along with skin clearance )+ Right Hemi scrotectomy and Tensor Fascia Lata flap reconstruction of skin defect .[Fig.1-5]

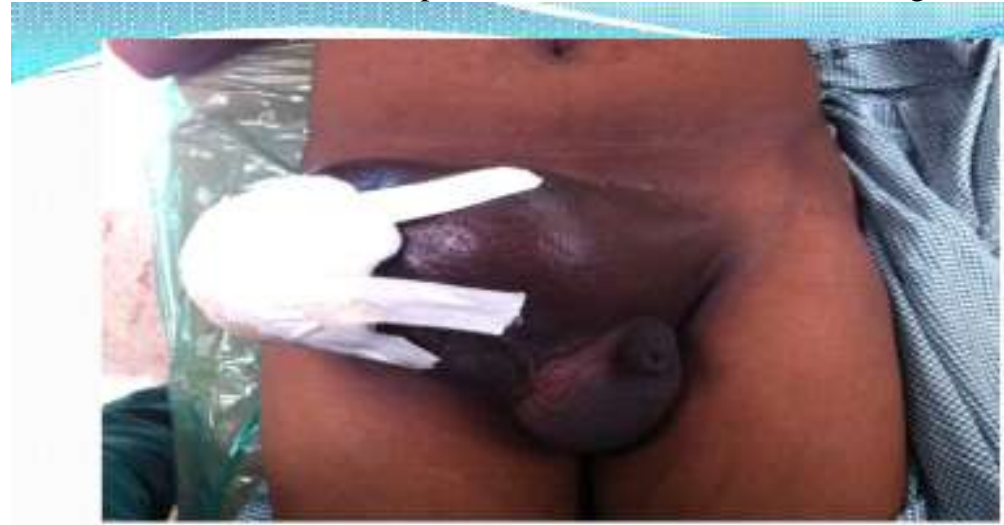

Fig.1-Right Inguinal nodal mass

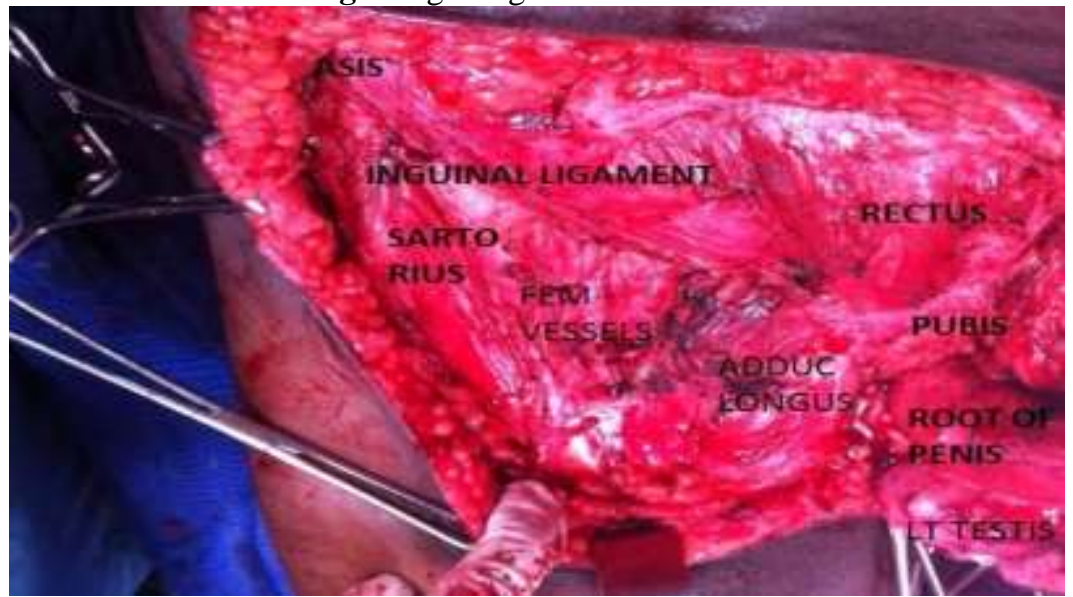

Fig.2: Post Radical Inguinal block dissection(RIBD) field 


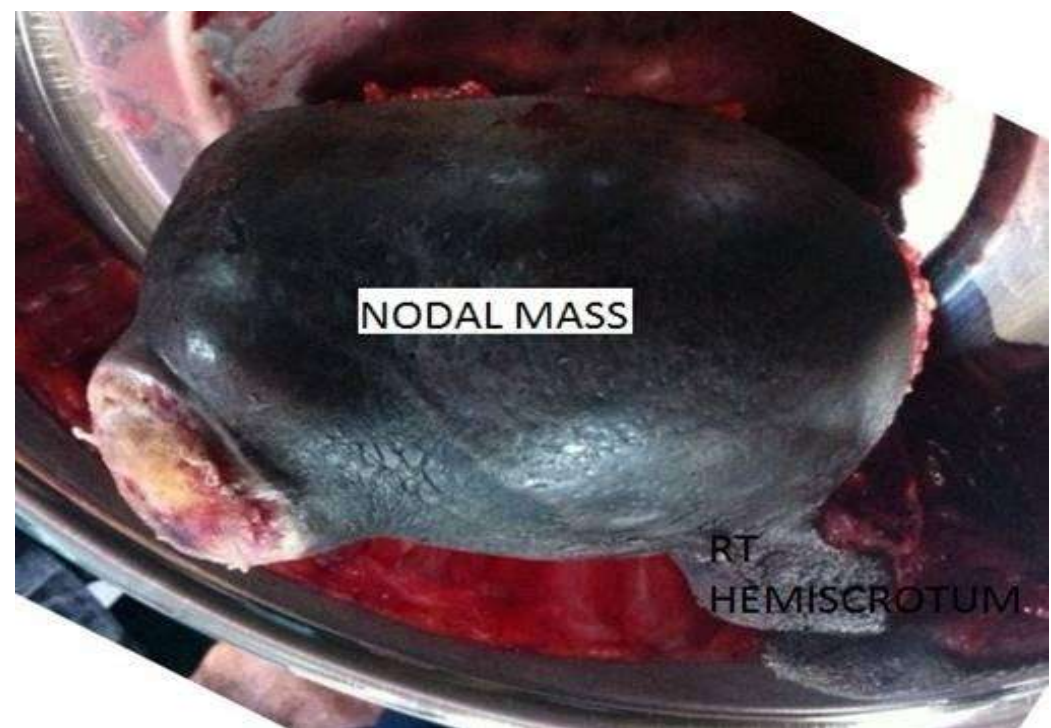

Fig.3: Specimen-Right RIBD with Right Hemiscrotectomy

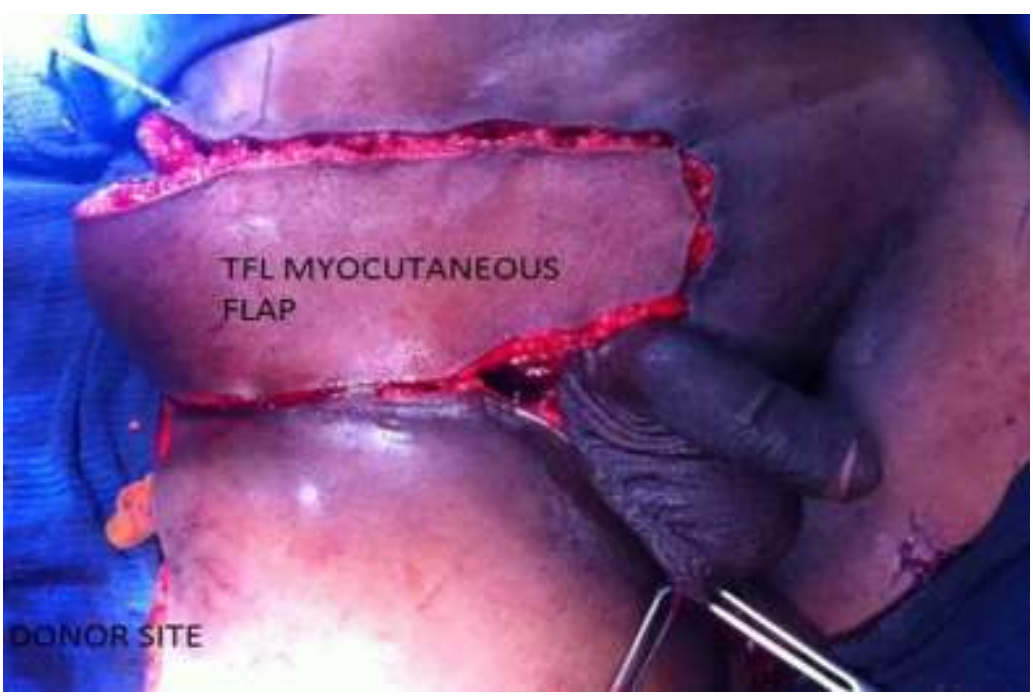

Fig.4: Tensor Fascia Lata Myocutaneous flap

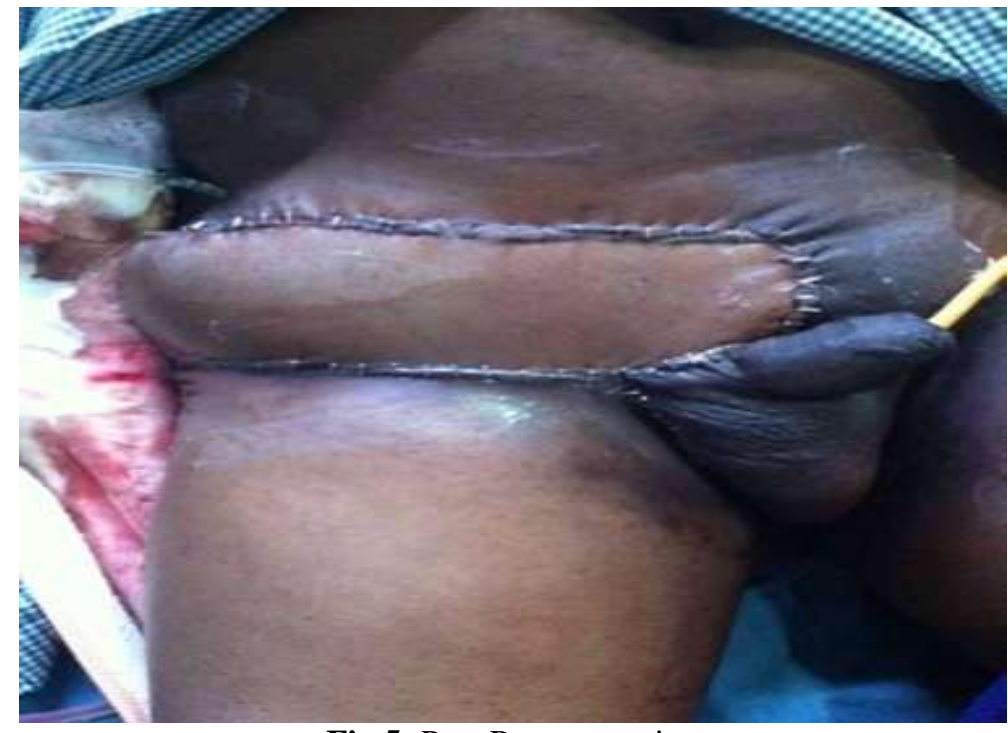

Fig.5: Post Reconstruction

Post op period: Uneventful recovery 
Histopathology: Microscopically, Lymph nodal mass entirely replaced by metastatic testicular tumour composed of immature ecto,endo and mesodermal components suggestive of Immature Teratoma.

Risk Classification - Intermediate (based on International Germ Cell Tumour Risk Classification)

Patient is under regular follow up clinically with observation of Serum Tumour markers 3 monthly for first 2 years, 6monthly for $3^{\text {rd }}$ to $5^{\text {th }}$ year and annually thereafter.

Current Status- Active Disease free survival of 1 year

\section{Discussion}

Testicular tumour spreads through lymphatic, hematogenous, direct extension and by local invasion. The lymphatic drainage of testis is retroperitoneal through gonadal vessels to para aortic and interaortocaval nodal basin. Inguinal nodes does not drain testis and if involved it becomes Metastatic disease (M1a). The possible mechanisms of inguinal nodal metastasis from a testicular tumour are as follows

i) Retrograde spread from bulky Retroperitoneal nodes,

ii) Breach of scrotal barrier eg.Scrotal orchidectomy, Past surgery in inguinoscrotal region,

iii) Aberrant lymphatics draining testis to inguinal nodes,

iv) Skip metastasis (by hematogenous route),

v) Tumour spillage or contamination during radical orchiectomy.

The incidence of inguinal lymph nodal metastasis was studied retrospectively in 695 patients with Stage I testicular cancer by Daugaard et al [1]. $2 \%$ of patients developed inguinal nodal metastasis. Nonseminomatous germ cell tumours more frequently invade inguinal lymph nodes than seminoma.

Testicular tumors in patients who have had the lymphatics disrupted by prior inguinal or scrotal surgery can metastasize to ipsilateral inguinal nodes primarily in addition to the usual retroperitoneal pattern. In patients with a prior history of orchidopexy or scrotal surgery who have a testicular tumor, the incidence of inguinal metastases has been reported in series varying from two percent up to 10 percent by Batata et al[2] .

In another retrospective series by Capelouto et al [3] in which trans scrotal approaches had been used for testicular tumour, a statistically significant increase in local recurrence rates compared with the recurrence rates when the inguinal approach was used $(2.9 \%$ vs. $0.4 \%)$. High ligation of the spermatic cord is therefore important.

For non-seminomatous testicular tumor with a previous history of scrotal and inguinal procedures Wheeler et al[4] advocated ipsilateral inguinal and bilateral retroperitoneal node dissection as the primary therapy.

The complications of inguinal nodal metastasis from a testicular tumour include upstaging of disease, local morbidity, erosion into femoral vessels, deep vein thrombosis and distal limb lymphedema.

In our case, surgery was contemplated because local morbidities are taken care of well by surgery, it offers disease free state by eradication of sanctuary sites of tumour and complete response to therapy is durable. The residual nodal mass contained 50\% viable tumour of which $30 \%$ was teratomatous component. Teratomatous component has the potential to grow by local extension and can cause vascular erosion. Although Teratoma per se doesn't have metastatic potential it can de differentiate and undergo malignant transformation like Sarcomas. Teratomatous component respond to Surgery rather chemo or radiotherapy.

This case report is presented in view of rarity of inguinal nodal metastasis from a testicular tumour in the absence of breach of scrotal barrier and other known causes. A similar case in which a true hermaphrodite with testicular seminoma with resulting metastases to the inguinal lymph nodes after radical orchidectomy has been reported by Mohamed Ismail et al [5]. To our best possible literary review our case report could be second such case.

\section{Acknowledgement}

We would like to thank Dr Akshaya D Baker and Dr Sharath kumar in helping with patient care and Dr Deepika Chandrasekaran in preparing the manuscript.

\section{References}

[1]. Daugaard G, Karas V, Sommer P: Inguinal metastases from testicular cancer. BJU Int. 2006, 97: 724-726. 10.1111/j.1464410X.2006.06017.x.View ArticlePubMedGoogle Scholar

[2]. Batata MA, Whitmore WF, Chu FC, Hilaris BS, Loh J, Grabstald H, Golbey R: Cryptorchidism and testicular cancer. J Urol. 1980, 124: 382-387.PubMedGoogle Scholar

[3]. Capelouto CC, J Urol., 1995 Mar;153(3 Pt 2):981-5 PMID: 7853587

[4]. Wheeler JS, Babayan RK, Hong WK, Krane RJ: Inguinal node metastases from testicular tumors in patients with prior orchiopexy. J Urol. 1983, 129: 1245-1247.PubMedGoogle Scholar

[5]. Mohamed Ismail, J Med Case Reports. 2010; 4: 378,Published online 2010 PMCID: PMC3003676. 